\title{
Relative Risk of Prolonged Operative Times From Inconsistent Surgical Teams
}

\author{
Franklin Dexter
}

Published online: 5 February 2015

(C) Société Internationale de Chirurgie 2015

Xiao et al. studied associations between heterogeneous teams of a surgeon, an operating room nurse, and a surgical technologist and several outcomes, including "prolonged operative time (in the longest quartile)" [1]. "Inconsistent teams were associated with" greater odds of "prolonged operative time $[1.52,95 \%$ confidence interval (CI) 1.20-1.91]."

From the authors' Fig. 3, when "both the circulating nurse and surgical technologist ... were among the three most frequent working with the surgeon," the "operative time (was) in the longest quartile" for $21 \%$ of cases. In contrast, when both rarely worked with the surgeon, $29 \%$ of cases were in the longest quartile. From the Discussion, "there was a $52 \%$ increase in occurrences of prolonged operative time." However, the relative risk would be approximately $1.38=29 \% / 21 \%$. The 1.52 is the odds ratio, not the relative risk. Is this interpretation correct?

The relative risk is needed for evaluating managerial importance and designing future studies. The odds ratio is a biased estimator for relative risk when incidences are not small (e.g., $>10.0 \%$ ) [2]. What is the estimate for the adjusted relative risk and its confidence interval $[3,4]$ ?

From the Introduction, it is stated that there is "higher likelihood of prolonged operative time." Knowing the likelihood ratio would indeed be helpful for evaluating the quantitative contribution of teams to the problem of prolonged operative times. To perform the arithmetic, Fig. 3 provides the incidences for consistent versus ad hoc teams. From Fig. 1, $39 \%$ of teams were consistent. The percentage of teams that were ad hoc, as needed to calculate the likelihood ratio, seems unclear. What was the likelihood ratio?

\section{References}

1. Xiao Y, Jones A, Zhang B et al (2015) Team consistency and occurrences of prolonged operative time, prolonged hospital stay, and hospital readmission: a retrospective analysis. World J Surg. doi:10.1007/s00268-014-2866-7

2. Prasad K, Jaeschke R, Wyer P et al (1998) Tips for teachers of evidence-based medicine: understanding odds ratios and their relationship to risk ratios. J Gen Intern Med 23:635-640

3. Zhang J, Yu KF (1998) What's the relative risk? A method of correcting the odds ratio in cohort studies of common outcomes. JAMA 280:1690-1691

4. Zou G (2004) A modified Poisson regression approach to prospective studies with binary data. Am J Epidemiol 159:702-706
F. Dexter $(\bowtie)$

Division of Management Consulting, Department of Anesthesia,

University of Iowa, Iowa City, IA, USA

e-mail: franklin-dexter@uiowa.edu 